\title{
Linha de Cuidado do Câncer do Colo do Útero no Amazonas: uma Análise da Prevenção ao Tratamento de Lesões Precursoras
}

\author{
doi: https://doi.org/10.32635/2176-9745.RBC.2021v67n3.1282
}

\author{
The Cervical Cancer Care Line in Amazonas State: an Analysis from Prevention to Treatment of Precursor Lesions \\ Línea de Atención del Cáncer de Cuello Uterino en el Amazonas: un Análisis de la Prevención al Tratamiento de las \\ Lesiones Precursoras
}

Gabriela Amaral de Sousa'; Juliana Nascimento Viana²; Celsa da Silva Moura Souza ${ }^{3}$; Rosana Pimentel Correia Moysés ${ }^{4}$

RESUMO

Introdução: $\mathrm{O}$ câncer do colo do útero tem alta morbimortalidade no Amazonas, demonstrando a importância da consolidação de uma linha de cuidado que busque a melhor utilização dos recursos em açôes de promoçáo, prevenção, detecção precoce e tratamento. Objetivo: Traçar o panorama dessa linha de cuidado no Amazonas, avaliar os indicadores de rastreio e diagnóstico do câncer do colo do útero e comparar o total de procedimentos realizados com o preconizado para a efetividade da linha de cuidado no período de 2016 a 2019. Método: Estudo descritivo, de abordagem quantitativa, que analisou a realização de procedimentos da linha de cuidado do câncer do colo do útero no Amazonas a partir de dados secundários do Sistema de Informaçáo do Programa Nacional de Imunizaçóes; do Sistema de Informaçóes Ambulatoriais do Sistema Único de Saúde, da Agência Nacional de Saúde Suplementar e do Instituto Brasileiro de Geografia e Estatística. Resultados: As análises demonstraram que a cobertura vacinal preconizada (80\%) não foi atingida em nenhum ano no período analisado. A quantidade de procedimentos realizados náo alcançou os parâmetros preconizados, mesmo nos anos em que se obteve melhores resultados: exames citopatológicos (-47,20\%) em 2017, colposcopias (-83,14\%) em 2016 e biópsias (-63,60\%) em 2019. Conclusáo: Os resultados obtidos demostram falhas na estratégia ao longo de toda a trajetória da linha de cuidado e nenhuma variável analisada atingiu seu objetivo, reforçando a necessidade de investimento em estratégias mais eficientes de prevenção e que tornem a linha de cuidado mais organizada.

Palavras-chave: Neoplasias do Colo do Útero/prevenção \& controle; Neoplasias do Colo do Útero/diagnóstico; Assistência Integral à Saúde; Saúde da Mulher; Brasil.

\begin{abstract}
Introduction: Cervical cancer has high morbidity and mortality in Amazonas State, which shows the importance of consolidating a care line that seeks the best use of resources in actions of promotion, prevention, early detection and treatment. Objective: Draw a panorama of this care line in Amazonas State, evaluate the indicators of screening and diagnosis of cervical cancer and compare the total number of performed procedures with the recommendations for cancer care line effectiveness from 2016 to 2019. Method: Descriptive quantitative approach study analyzing the performance of procedures of the cervical cancer care line in Amazonas State, based on secondary data of the National Immunization Program Information System of the Outpatient and Hospital Information Systems of the SUS - National Health System, of the National Supplementary Health Agency and of the Brazilian Institute of Geography and Statistics of. Results: The analyses demonstrated that the vaccination goals (80\%) were not achieved in the period investigated. The number of procedures performed did not reach the recommended parameters, even in the years when the best results were obtained: cytopathological exams $(-47.20 \%)$ in 2017, colposcopies (-83.14\%) in 2016 and biopsies (-63.60\%) in 2019. Conclusion: The results obtained reveal flaws in the strategy throughout the entire trajectory of the care line, none of the parameters analyzed reached its goal, reinforcing the need to invest in more efficient prevention strategies to make the care line more organized.

Key words: Uterine Cervical Neoplasms/prevention \& control; Uterine Cervical Neoplasms/diagnosis; Comprehensive Health Care; Women's Health; Brazil.
\end{abstract}

\section{RESUMEN}

Introducción: El cáncer de cuello uterino tiene una alta morbilidad y mortalidad en el Amazonas, lo que demuestra la importancia de consolidar una línea de atención que busque el mejor uso de los recursos en acciones de promoción, prevención, detección temprana y tratamiento. Objetivo: Trazar el panorama de esta línea de atención en el Amazonas, evaluar los indicadores de detección y diagnóstico del cáncer de cuello uterino y comparar el número total de procedimientos realizados con el recomendado para la efectividad de la línea de atención, desde 2016 hasta 2019. Método: Estudio descriptivo con un enfoque cuantitativo que analizó la producción de procedimientos de la línea de atención del cáncer de cuello uterino en el Amazonas, con base en datos secundarios del Sistema de Información del Programa Nacional de Inmunizaciones; el Sistema de Información Ambulatoria del Sistema Único de Salud, Organismo nacional de Salud Suplementaria y el Instituto Brasileño de Geografía y Estadística. Resultados: Los análisis mostraron que la cobertura de vacunación recomendada (80\%) no se alcanzó en ningún año del período analizado. El número de procedimientos realizados no alcanzó los parámetros recomendados, incluso en los ańos en que se obtuvieron los mejores resultados: exámenes citopatológicos $(-47,20 \%)$ en 2017 , colposcopias $(-83,14 \%)$ en 2016 y biopsias $(-63,60 \%)$ en 2019 . Conclusión: Los resultados obtenidos muestran fallas en la estrategia a lo largo de toda la trayectoria de la línea de cuidados, en ninguna variable analizada se lograron las metas, reforzando la necesidad de invertir en estrategias de prevención más eficientes que hagan más organizada la línea de cuidados.

Palabras clave: Neoplasias del Cuello Uterino/prevención \& control; Neoplasias del Cuello Uterino/diagnóstico; Atención Integral de Salud; Salud de la Mujer; Brasil.

\footnotetext{
1-4Universidade Federal do Amazonas. Faculdade de Medicina. Núcleo de Atividades Integradas. Manaus (AM), Brasil. E-mails: gabrielaamaral.med@gmail.com; juliana.nviana@live.com; celsa22@hotmail.com; rosanamoyses@ufam.edu.br/ rosanapcsmsdc@gmail.com. Orcid iD: https://orcid.org/0000-0002-7642-3494; Orcid iD: https://orcid.org/0000-0003-3014-7495; Orcid iD: https://orcid.org/0000-0002-1669-1462; Orcid iD: https://orcid.org/0000-0003-4992-1698 Endereço para correspondência: Gabriela Amaral de Sousa. Av. General Rodrigo Octavio Jordão Ramos, 1200 - Coroado I. Manaus (AM), Brasil. CEP 69067-005. E-mail: gabrielaamaral.med@gmail.com
} 


\section{INTRODUÇÃO}

O câncer do colo do útero é uma doença com grande impacto na saúde da mulher no mundo. No Brasil, ainda existe alta morbimortalidade pelo câncer do colo do útero, apresentando os piores indicadores na Regiáo Nordeste e Norte do país. Os Estados do Pará e Amazonas apresentam os dados mais alarmantes de toda Regiáo. No período de 2012 a 2018, foram registrados no Sistema de Informaçáo de Mortalidade (SIM) 1.926 óbitos por câncer do colo do útero no Estado do Amazonas. Além disso, somente no ano de 2019, foram registradas 194 mortes por câncer do colo do útero no hospital de referência Fundação Centro de Controle do Câncer do Amazonas ${ }^{1,2}$.

Diante desse panorama de alta carga de doença, fica clara a necessidade da concretizaçáo de uma linha de cuidado para o câncer do colo do útero no Amazonas, buscando a melhor utilização dos recursos em ações de promoção, prevenção, detecção precoce e tratamento. Afinal, a linha de cuidado busca "o estabelecimento do percurso assistencial organizando o fluxo dos indivíduos, de acordo com suas necessidades", tendo como objetivo principal $o$ atendimento integral em saúde ${ }^{3}$.

Hoje, no Brasil, pode-se afirmar que a linha de cuidado do câncer do colo do útero se inicia na prevenção primária, por meio da vacinação contra o papilomavírus humano (HPV) para meninos e meninas, seguida da prevençáo secundária, que é a realização do rastreio da doença pelo exame citopatológico do colo do útero (Papanicolaou). A partir dos resultados desse exame, passa-se para novas etapas de investigação diagnóstica e tratamento de lesōes precursoras. Em caso de diagnóstico de malignidade, encaminha-se o paciente para o tratamento da doença na alta complexidade, podendo chegar aos cuidados paliativos ${ }^{4}$.

A literatura comprova que a consolidação da linha de cuidado por meio de redes de atenção organizadas por território, apesar de ainda ser um desafio para o Brasil, é a melhor estratégia de estruturação de política públicas para atendimento integral da população. Para analisar a efetividade das açóes da linha de cuidado, os sistemas de informação do Sistema Único de Saúde (SUS) podem ser importantes instrumentos, pois permitem traçar o panorama anual da assistência à saúde ${ }^{5-7}$.

Sendo assim, este estudo pretende analisar os registros de procedimentos realizados da linha de cuidado do câncer do colo do útero no Estado do Amazonas, considerando a prevenção ao tratamento de lesóes precursoras no período de 2016 a 2019, de forma a comparar a necessidade anual pactuada e a realizaçáo dos procedimentos dessa linha de cuidado.

\section{MÉTODO}

Trata-se de um estudo descritivo, com abordagem quantitativa, a partir das bases de registro de dados, sem identificação de indivíduos, do Sistema de Informação do Programa Nacional de Imunizaçôes (SI-PNI) ${ }^{5}$, do Sistema de Informaçóes Ambulatoriais do Sistema Único de Saúde (SIA/SUS) ${ }^{8}$, do Instituto Brasileiro de Geografia e Estatística (IBGE) e da Agência Nacional de Saúde Suplementar (ANS).

A população foi constituída de meninas de 9 a 14 anos, meninos de 10 a 14 anos e mulheres de 25 a 64 anos, no ano de 2016, com dados coletados no site do IBGE? Os dados referentes à vacinação contra o HPV foram coletados no SI-PNI ${ }^{5}$. Já os dados sobre os procedimentos de rastreio, investigação diagnóstica e tratamento de lesóes precursoras foram coletados no SIA/SUS ${ }^{8}$. Para análise das faixas etárias da vacinação, foram consideradas as diretrizes para vacinaçáo contra HPV vigentes em cada período: em 2016, 9-13 anos para meninas e 10-13 para os meninos; de 2017 a 2019, de 9-14 para meninas e 11-14 para os meninos. Para análise dos demais procedimentos da linha de cuidado, a faixa etária foi de 25-29, 30-34, 35-39, 40-49, 50-59, 60-64, 5,10 .

Os indicadores de prevenção primária descritos no SI-PNI (2016 a 2019) foram: 1. Dados referentes à vacinação do HPV quadrivalente feminino de 9 a 13 anos (códigos do 067 ao 071) e HPV quadrivalente masculino de 10 a 13 anos (códigos do 108 ao 111/códigos do 081 ao 086) nos anos de 2016 e 2017; 2. Dados referentes à vacinação do HPV quadrivalente feminino de 9 a 14 anos (códigos do 067 ao 071) e HPV quadrivalente masculino de 11 a 14 anos (códigos do 108 ao 111/códigos do 081 ao 086) nos anos de 2018 e 2019.

Os indicadores de rastreio descritos no SIA/SUS (2016 a 2019) foram: (1) exame citopatológico cervicovaginal/ microflora - rastreamento (código 0203010086); e (2) exame citopatológico cervicovaginal/microflora (código 0203010019).

Os indicadores de investigação diagnóstica descritos no SIA/SUS (2016 a 2019) foram: (1) colposcopia (código 021104002-9); e (2) biópsia do colo uterino (código 0201010666).

Os indicadores de tratamento de lesôes precursoras descritos no SIA/SUS (2016 a 2019) foram: (1) excisão tipo 1 do colo uterino (código 0409060089); (2) excisão tipo 2 do colo uterino (código 0409060305); e (3) excisão tipo 3 do colo uterino (código 0409060038).

Quanto à análise dos dados, primeiramente os dados foram descritos e organizados no software Excel $^{\circ}$, utilizando a planilha eletrônica para armazenamento e o programa IBM Statistical Package for the Social Science (SPSS), versão 22.0. 
Para o cálculo da prevenção primária, realizou-se a análise descritiva (média e frequência) das coberturas vacinais contra o HPV por dose e sexo, considerando a meta de $80 \%$ de cobertura nas faixas etárias definidas pelo Programa Nacional de Imunização $0^{4,10}$.

Foi utilizada como referência a população feminina do Estado do Amazonas de 25 a 64, seguindo as recomendaçóes da Diretrizes Brasileiras para o Rastreio de Câncer do Colo do Útero, de acordo com a projeção populacional do IBGE para cada ano analisado. Contudo, no intuito de náo superestimar os atendimentos realizados nos estabelecimentos de saúdes públicos ou conveniados ao SUS, foi realizada uma estimativa de mulheres usuárias desse Sistema, subtraindo-se da população total da faixa etária alvo ( 25 a 64 anos) o número correspondente à proporção de mulheres atendidas por planos de saúde, de acordo com os dados da ANS, no Estado do Amazonas ${ }^{11,12}$.

Além disso, foram utilizados os parâmetros técnicos para o rastreamento do câncer do colo do útero, que estimam o número de procedimentos necessários, segundo a população observada, para rastrear e seguir $100 \%$ de mulheres da população-alvo mimetizando um cenário fictício com rastreio organizado, possibilitando a comparação com o cenário real atual do país, cujo rastreio é oportunístico e com diferentes desafios nas açôes de continuidade do cuidado ${ }^{13}$.

Os percentuais de déficit ou excesso de procedimentos para rastreamento, investigação diagnóstica e tratamento de lesōes precursoras do câncer do colo do útero foram calculados por meio da seguinte fórmula:

$n^{0}$. de procedimentos realizados $-n^{0}$. de procedimentos necessários $x 100$

\section{$\mathrm{n}^{0}$. de procedimentos necessários}

A fórmula, descrita anteriormente, estimou ainda a cobertura anual de exames citopatológicos do colo do útero em mulheres da população-alvo. Foi calculada ainda a cobertura anual de exames citopatológicos do colo do útero em mulheres da população-alvo de 25 a 64 anos. $\mathrm{O}$ resultado desse cálculo é um indicador de processo descrito na ficha técnica de indicadores das açôes de controle do câncer do colo do útero do Instituto Nacional de Câncer José Alencar Gomes da Silva (INCA) ${ }^{14}$.

Esse indicador de processo é estabelecido pelo poder público e avalia o quanto da população-alvo está sendo alcançada, em um determinado local e ano, pelas açôes de prevenção do câncer do colo do útero, por meio do rastreamento, pela divisão do número de exames citopatológicos do colo do útero e do número de mulheres na faixa etária alvo, no respectivo ano e local ${ }^{14}$.
Este estudo não foi submetido ao Comitê de Ética em Pesquisa por se tratar de pesquisa que utilizou banco de dados públicos, respeitando o disposto no Art.1, Inciso III, da Resolução 510/2016 do Conselho Nacional de Saúde ${ }^{15}$, que dispôe sobre as diretrizes e normas regulamentadoras de pesquisa envolvendo seres humanos.

\section{RESULTADOS}

No que diz respeito à vacinação para o HPV, a cobertura mais expressiva para meninas e meninos, 19,6\% e $39,2 \%$, respectivamente, foi alcançada na Dose $1 \mathrm{em}$ 2017. No entanto, a cobertura ideal preconizada (80\%) não foi atingida em nenhuma dose, considerando ambos os sexos, nos anos do período analisado (Figura 1).

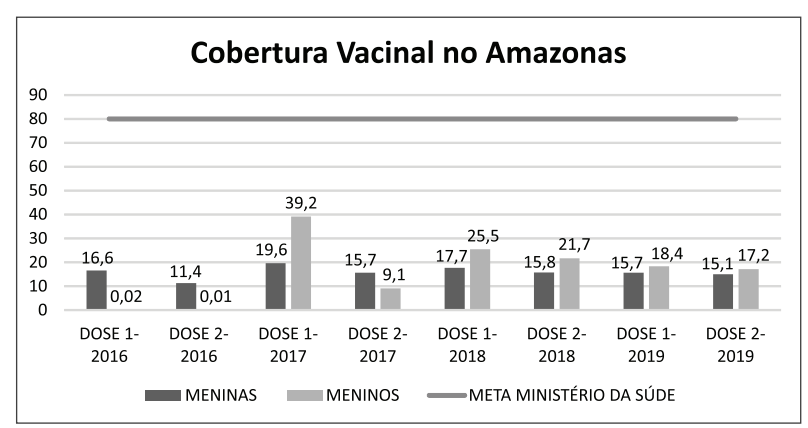

Figura 1. Cobertura vacinal no Estado do Amazonas entre 2016 e 2019

No Estado do Amazonas, entre 2016 e 2019, em média $83 \%$ da população feminina, na faixa etária de 25 a 64 anos, dependia do SUS, conforme descrito na Figura 2.

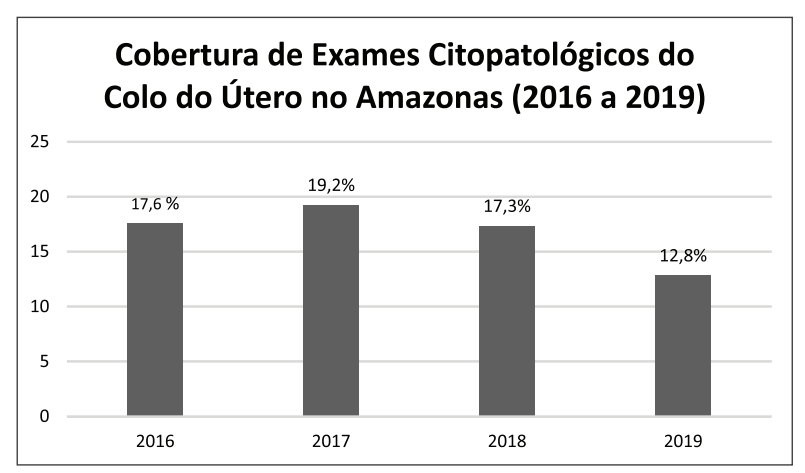

Figura 2. População feminina usuária do SUS

Com base nos parâmetros técnicos para o rastreamento do câncer do colo do útero, a quantidade de procedimentos, que seriam realizados em um cenário ideal, está representada em valores absolutos (Tabela 1 ). Nesse panorama, $100 \%$ das mulheres na faixa etária seriam rastreadas e receberiam seguimento adequado quando necessário. A quantidade em valores absolutos de exames, 
que foram realizados realmente no período analisado, também foi descrita, além dos percentuais de déficit dos procedimentos analisados, obtidos pela fórmula apresentada nos métodos deste estudo ${ }^{13}$.

Tabela 1. Comparação entre a necessidade anual estimada e a oferta de exames citopatológicos, colposcopias e biópsias entre os anos de 2016 e 2019

\begin{tabular}{c|c|c}
\hline \multicolumn{3}{c}{ EXAMES CITOPATOLÓGICOS } \\
\hline & Necessários & Realizados (diferença em \%) \\
\hline 2016 & $325.605,74$ & $157.754,00(-51,55)$ \\
\hline 2017 & $336.164,60$ & $177.500,00(-47,20)$ \\
\hline 2018 & $350.126,22$ & $164.352,00(-53,06)$ \\
\hline 2019 & $360.866,33$ & $125.018,00(-65,36)$ \\
\hline \multicolumn{2}{|c}{ COLPOSCOPIAS } \\
\hline & Necessários & Realizados (diferença em \%) \\
\hline 2016 & $14.028,37$ & $2.365,00(-83,14)$ \\
\hline 2017 & $14.483,28$ & $2.090,00(-85,57)$ \\
\hline 2018 & $15.084,80$ & $2.209,00(-85,36)$ \\
\hline 2019 & $15.547,53$ & $2.211,00(-85,78)$ \\
\hline & BIÓPSIAS DO COLO UTERINO \\
\hline & Necessários & Realizados (diferença em \%) \\
\hline 2016 & $2.067,34$ & $384,00(-81,43)$ \\
\hline 2017 & $2.134,38$ & $674,00(-68,42)$ \\
\hline 2018 & $2.223,02$ & $661,00(-70,27)$ \\
\hline 2019 & $2.291,21$ & $834,00(-63,60)$ \\
\hline
\end{tabular}

Em 2019, a produção de exames citopatológicos realizados comparada à necessária foi a que apresentou maior déficit $(-65,36 \%)$. Em todos os anos analisados, o número de colposcopias realizadas foram inferiores ao necessário. Esse cenário se repete no que diz respeito a biópsias do colo do útero, com valores particularmente baixos em 2016, cujo déficit chegou a $81 \%$.

A cobertura anual de exames citopatológicos, no Estado do Amazonas, durante o período analisado, não foi alcançada; 2017 foi o ano em que o Estado apresentou melhor desempenho, contudo, ainda correspondia a menos de $20 \%$ da população-alvo (Figura 3).

\section{DISCUSSÃO}

Uma linha de cuidado deve abranger açôes e serviços de saúde embasados em critérios epidemiológicos e de regionalização que garantam acesso aos serviços e ao cuidado integral, além de estabelecer um "percurso" no intuito de organizar o fluxo de pacientes de acordo com suas necessidades, promovendo a prevenção, acesso ao rastreamento, diagnóstico precoce e tratamento adequado ${ }^{16}$.

\section{Média da População Feminina de 25 a 64 Anos (2016 a 2019)}

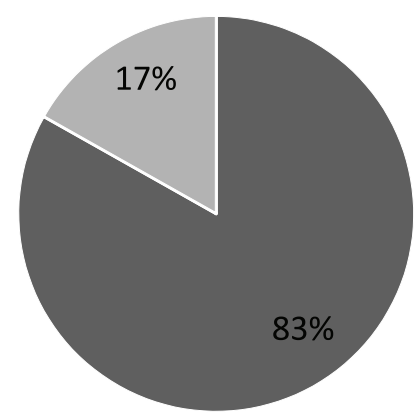

- USUÁRIAS SUS = USUÁRIAS SAÚDE COMPLEMENTAR

Figura 3. Cobertura de exames citopatológicos do colo do útero no Estado do Amazonas entre os anos de 2016 e 2019

Atualmente, o fluxo para essa doença abrange prevenção primária por meio da vacinação e do rastreio estabelecido nas Diretrizes Brasileiras para o Rastreio do Câncer do Colo do Útero ${ }^{11}$. A imunização contra o HPV se inicia na pré-adolescência para meninos e meninas. Em seguida, em certo momento, é realizado o rastreio oportunístico de todas as mulheres elegíveis por meio do exame citopatológico de material cervicovaginal. Destas, para um pequeno grupo cujos exames mostram-se alterados, é dado o seguimento necessário de acordo com o grau de lesão encontrada. Posteriormente, os exames diagnósticos de colposcopia e biópsia são utilizados para definir condutas. Por fim, para as mulheres com lesóes invasivas, a última parte da linha de cuidado será o tratamento adequado, podendo ser composto de cirurgia, quimioterapia, radioterapia ou da combinação destas ${ }^{11}$.

O Estado do Amazonas foi o primeiro no país a disponibilizar a vacina contra HPV no SUS a partir do ano de 2013. No restante do país, ela foi implementada como prevenção primária em 2014; no entanto, esse processo foi feito de maneira gradativa. Dessa forma, ao longo dos anos, houve mudanças na faixa etária contemplada, bem como na quantidade e no intervalo entre as doses. No primeiro momento, era oferecida para meninas entre 11 e 13 anos de idade em três doses (zero, seis e 60 meses). Em 2015 e 2016, houve ampliação da faixa etária para 9 a 13 anos e modificação do esquema para apenas duas doses (0 e 6 meses), respectivamente. A partir de 2017, ampliou-se a vacinação para as meninas de 9 a 14 anos e introduziu-se oficialmente para os meninos de 11 a 14 anos $^{17,18}$.

Todas essas adaptaçôes foram necessárias e embasadas em estudos científicos para garantir a eficácia dessa medida profilática, uma vez que a vacina é um método eficaz e com excelente custo-benefício para diminuir o grave desfecho que é o câncer do colo do útero. Entretanto, para garantir 
esses benefícios e a vacinação ser considerada eficiente, é necessário que a cobertura vacinal atinja pelo menos $80 \%$ da população-alvo ${ }^{19,20}$.

O Estado do Amazonas não atingiu o preconizado segundo a dose e o sexo em nenhum ano entre o período de 2016 e 2019. As maiores taxas foram observadas em 2017, também sendo esse o único ano em que o percentual de cobertura foi maior no sexo feminino. É interessante notar que, nos demais anos analisados, a cobertura foi maior em meninos; esse dado também relatado em outros estudos pode ser atribuído à recente inclusão desse grupo no calendário vacinal do SUS contra o $\mathrm{HPV}^{17}$.

É importante observar que as taxas se mantiveram abaixo do preconizado em ambos os sexos, ressaltando a necessidade de estratégias de sensibilização para a adesão à vacinação contra o HPV. Infelizmente, essa realidade não é exclusividade dessa unidade da federação e, em razão desse cenário preocupante, várias pesquisas foram realizadas no intuito de identificar os fatores que prejudicam a adesão, sendo a falta de informação sobre a segurança e a eficácia da vacina para os adolescentes e seus responsáveis a dificuldade de acesso à vacinação, e os eventos adversos pós-vacinação, os motivos frequentemente apontados ${ }^{21,22}$.

No que diz respeito ao rastreio do câncer do colo do útero e suas lesôes precursoras, o principal método utilizado atualmente é a realização periódica do exame citopatológico do colo uterino, sendo este um teste capaz de detectar alteraçóes celulares nessa região. Esse exame deve ser realizado em mulheres que tenham entre 25 e 64 anos, sendo os dois primeiros exames feitos com intervalo anual e, se ambos não apresentarem nenhuma alteração, os próximos devem ser realizados a cada três anos ${ }^{11,16}$.

No Amazonas, para que se cumprisse o preconizado para rastrear efetivamente a população, seria necessário a realizaçáo de cerca de 343 mil exames citopatológicos do colo uterino por ano entre 2016 e 2019, levando em consideração a populaçáo-alvo. Porém, mesmo no ano em que se obteve a melhor taxa de produção de exames, foram realizados pouco mais de 177 mil exames. Estudos descrevem que a efetividade do rastreamento pode estar associada à estratégia adotada. Apesar de algumas Regióes do Brasil apresentarem lenta tendência decrescente das taxas de câncer do colo do útero, o rastreamento de caráter oportunístico realizado no país e consequentemente no Estado do Amazonas possui limitaçôes. A estratégia de rastreio não oportunístico; ou seja, assegurar um mecanismo de recrutamento da população-alvo, tem sido apontada como uma excelente alternativa para mudar essa realidade, possibilitando melhor relação custo-benefício com alta cobertura populacional ${ }^{23,24}$.

A mesma situação de baixa cobertura de exames citopatológicos encontrada no Estado do Amazonas já foi relatada em outros estudos e é preocupante, pois atingir os níveis preconizados é indispensável para se obter significativa redução da incidência e da mortalidade. Em países que alcançaram cobertura superior a $70 \%$, as taxas de mortalidade foram inferiores ou iguais a duas mortes por 100 mil mulheres por ano ${ }^{11,25}$.

Observa-se grande déficit também quanto aos indicadores de investigação diagnóstica, colposcopia e biópsia, cujos valores projetados são maiores do que os observados na prática, com base na população total do Estado, dentro da faixa-etária alvo. Essa carência ressalta a dificuldade de organização da rede assistencial e pode estar estritamente relacionada às altas taxas de incidência e mortalidade por câncer do colo útero no Amazonas ${ }^{26}$.

Em relação aos procedimentos de tratamento de lesōes precursoras, foram consideradas as excisões dos tipos 1, 2 e 3 do colo uterino; no entanto, na base SIA/SUS ${ }^{8}$, não constavam registros de dados que possibilitassem uma análise robustas dessas variáveis. Essa limitação pode estar associada à lógica contábil herdada pelo sistema que o precedia, o qual se baseava na antiga Guia de Autorização de Pagamento (GAP). Contudo, ao longo dos anos, aconteceram melhorias no intuito de garantir informações mais fidedignas referentes aos atendimentos ambulatoriais; logo, o SIA/SUS constitui um considerável instrumento para avaliação, controle e planejamento, sobretudo da assistência médica fornecida pelo SUS $8,27,28$.

Este estudo, apesar das limitaçóes, por utilizar dados secundários, apresenta resultados que reforçam um cenário preocupante e a necessidade de investir em estratégias mais eficientes de prevenção e que impactem na diminuição da morbimortalidade por essa doença no Estado do Amazonas.

\section{CONCLUSÃO}

As metas de vacinação e cobertura anual de exames citopatológicos do colo do útero não foram atingidas no Estado do Amazonas em nenhuma dose, em nenhum sexo e em nenhuma faixa etária, no período analisado. Bem como, ao comparar a necessidade de procedimentos para rastrear e seguir $100 \%$ de mulheres da população-alvo com a quantidade de procedimentos realizados atualmente, houve déficit em todos os parâmetros analisados. Esses resultados ressaltam as falhas ao longo de toda a trajetória da linha de cuidado dessa doença. Os desafios para concretização dessa linha de cuidado precisam ser analisados e corrigidos, na busca por melhores resultados na adesẫo ao rastreio, na investigação diagnóstica e no tratamento de lesóes precursoras do câncer do colo no Amazonas. 


\section{CONTRIBUIÇÕES}

Gabriela Amaral de Sousa, Juliana Nascimento Viana e Rosana Pimentel Correia Moysés contribuíram na concepção e/ou no planejamento do estudo; na obtenção, análise e interpretação dos dados; assim como na redação e revisão crítica. Celsa da Silva Moura Souza contribuiu na redação e revisão crítica. Todos os autores aprovaram a versão final a ser publicada.

\section{DECLARAÇÃO DE CONFLITO DE INTERESSES}

Nada a declarar.

\section{FONTES DE FINANCIAMENTO}

Conselho Nacional de Desenvolvimento Científico e Tecnológico (CNPQ). Programa Institucional de Bolsas de Iniciação Científica (PIBIC). Universidade Federal do Amazonas (Edital PIBIC2019/2020).

\section{REFERÊNCIAS}

1. Organização Pan-Americana da Saúde. Controle integral do câncer do colo do útero: guia de práticas essenciais [Internet]. Washington, DC: 2016 [acesso 2019 maio 10]. Disponível em: https://iris.paho.org/ bitstream/handle/10665.2/31403/9789275718797-por. pdf?sequence $=1$ \&isAllowed $=y$

2. Fundação Centro de Controle de Oncologia do Estado do Amazonas (BR). Relatório anual de gestão [Internet]. Manaus, AM: FCECON; 2019 [acesso 2020 jun 2]. Disponível em: http://www.fcecon.am.gov.br/ wp-content/uploads/2020/08/RELAT\%C3\%93RIOANUAL-DE-GEST\%C3\%83O-2019_compressed.pdf

3. Frigo J, Rodrigues RM, Ledra F, et al. A integralidade da atençáo e o câncer do colo do útero: uma revisão integrativa de literatura. UNINGÁ Review [Internet]. 2016 [acesso 2019 maio 11];27(3):36-43. Disponível em: http://revista.uninga.br/index.php/uningareviews/ article/view/1832/1432

4. Ministério da Saúde (BR), Secretaria de Vigilância em Saúde, Departamento de Vigilância das Doenças Transmissíveis, Coordenação Geral do Programa Nacional de Imunizaçóes. Guia prático sobre o HPV: perguntas e respostas. Brasília, DF: CGPNI; 2013 nov [acesso 2019 abr 22]. Disponível em: http:// drguilhermeleme.com.br/wp-content/uploads/2016/01/ Guia_Pratico_HPV_Perguntas_e_Respostas.pdf

5. SI-PNI: Sistema de Informaçôes do Programa Nacional de Imunizaçóes [Internet]. Versão 5.3.4. Brasília, DF: Secretaria de Atençáo à Saúde, Departamento de Vigilância das Doenças Transmissíveis. [data desconhecida] - [acesso 2019 abr 12]. Disponível em: http://sipni.datasus.gov.br/si-pni-web/faces/ apresentacaoSite.jsf\#

6. Paula SHB, Volochko A, Figueiredo R. Linha de cuidado de câncer de mama e de colo de útero: um estudo sobre referência e contrarreferência em cinco regióes de saúde de São Paulo, Brasil. BIS, Bol. Inst. Saúde [Internet]. 2016 dez [acesso 2019 abr 12];17(2):146-65. Disponível em: https://docs.bvsalud.org/biblioref/2019/10/1021674/ bis-v17n2-saude-e-direitos-sexuais-146-165.pdf

7. Melo ENN. Redes de atenção à saúde: atenção básica no cuidado à saúde da mulher [dissertação na Internet]. Goiás, GO: Universidade Federal de Goiás; 2016 [acesso 2019 maio 2]. 92 p. Disponível em: http://repositorio. bc.ufg.br/tede/handle/tede/6034

8. SIA/SUS: Sistema de Informação Ambulatoriais do SUS [Internet]. Brasília, DF: DATASUS. [2012] - . Produção ambulatorial do SUS - Amazonas; [acesso 2019 abr 12]. Disponível em: http://tabnet.datasus.gov.br/cgi/tabcgi. exe?sia/cnv/qbam.def

9. Instituto Brasileiro de Geografia e Estatística. Pesquisa de informações básicas municipais: perfil dos municípios brasileiros 2013 [Internet]. Rio de Janeiro: IBGE; 2014 [acesso 2019 abr 13]. Disponível em: https://biblioteca. ibge.gov.br/visualizacao/livros/liv86302.pdf

10. Ministério da Saúde (BR), Secretaria de Vigilância em Saúde, Departamento de Vigilância das Doenças Transmissíveis, Coordenação Geral do Programa Nacional de Imunizaçôes. Informe técnico da ampliação da oferta das vacinas papilomavírus humano 6, 11, 16 e 18 (recombinante): vacina HPV quadrivalente e meningocócica C (conjugada) [Internet]. Brasília, DF: CGPNI; 2018 mar [acesso 2019 abr 13]. Disponível em: https://portalarquivos2.saude.gov.br/images/pdf/2018/ marco/14/Informe-T--cnico-HPV-MENINGITE.pdf

11. Instituto Nacional de Câncer José Alencar Gomes da Silva. Diretrizes brasileiras para o rastreamento do câncer do colo do útero [Internet]. 2. ed. rev. ampl. atual. Rio de Janeiro: INCA; 2016 [acesso 2019 jul 18]. Disponível em: https://www.inca.gov.br/sites/ ufu.sti.inca.local/files//media/document//diretrizes paraorastreamentodocancerdocolodoutero_2016_ corrigido.pdf

12. ANS: Informaçóes em Saúde Suplementar [Internet]. Rio de Janeiro: Agência Nacional de Saúde Suplementar. [data desconhecida] - [acesso 2020 fev 5]. Disponível em: http://www.ans.gov.br/anstabnet/cgi-bin/dh?dados/ tabnet_cc.def

13. Instituto Nacional de Câncer José Alencar Gomes da Silva. Parâmetros técnicos para o rastreamento do câncer do colo do útero [Internet]. Rio de Janeiro: INCA; 2019 [acesso 2020 fev 7]. Disponível em: https://www.inca. gov.br/sites/ufu.sti.inca.local/files//media/document// parametros_tecnicos_colo_do_utero_2019.pdf

14. Instituto Nacional de Câncer José Alencar Gomes da Silva. Ficha técnica de indicadores das açôes de controle 
do câncer do colo do útero [Internet]. Rio de Janeiro: INCA; 2014 dez [acesso 2020 mar 20]. Disponível em: https:/www.inca.gov.br/sites/ufu.sti.inca.local/files// media/document//fichatecnicaindicadorescolo14.pdf

15. Conselho Nacional de Saúde (BR). Resolução no 510, de 7 de abril de 2016. Dispóe sobre as normas aplicáveis a pesquisas em Ciências Humanas e Sociais cujos procedimentos metodológicos envolvam a utilizaçáo de dados diretamente obtidos com os participantes ou de informaçóes identificáveis ou que possam acarretar riscos maiores do que os existentes na vida cotidiana, na forma definida nesta Resolução. Diário Oficial da Uniāo. 2016 maio 24; Seção I:44.

16. Ministério da Saúde (BR), Secretaria de Atenção à Saúde, Departamento de Atenção Básica. Controle dos cânceres do colo do útero e da mama [Internet]. 2. ed. Brasília, DF: Ministério da Saúde; 2013 [acesso 2020 fev 5]. Disponível em: http://bvsms.saude.gov.br/bvs/ publicacoes/controle_canceres_colo_utero_2013.pdf

17. Souza WM, Silva AD, Santos AD, et al. Análise da imunização contra o HPV no Brasil: um estudo ecológico exploratório de 2016 a 2018. Rev Eletr Ciên Tecnol Inova. 2020;1:1-9. doi: https://doi.org/10.9789/26754932.rectis.v1.9727

18. Moura LL. Cobertura vacinal contra o Papilomavírus Humano (HPV) em meninas e adolescentes no Brasil: análise por coortes de nascimentos [dissertação na Internet]. Rio de Janeiro: Fundação Oswaldo Cruz, Escola Nacional de Saúde Pública Sergio Arouca; 2019. 91 p. Disponível em: https://www.arca.fiocruz.br/handle/ icict/37391

19. Santos ACS, Silva NNT, Carneiro CM, et al. Knowledge about cervical cancer and HPV immunization dropout rate among Brazilian adolescent girls and their guardians. BMC Public Health. 2020;20(301). doi: https://doi. org/10.1186/s12889-020-8410-9

20. World Health Organization. WHO guide for standardization of economic evaluations of immunization programmes [Internet]. 2nd ed. Geneva: WHO; 2019 Oct [cited 2020 feb 7]. Available from: https://apps.who. int/iris/bitstream/handle/10665/329389/WHO-IVB19.10-eng.pdf?ua=1

21. Pereira IMR. Análise descritiva da cobertura da vacina HPV quadrivalente no Brasil, entre 2016 e 2017 [monografia na Internet]. Brasília, DF: Universidade de Brasília; 2018. 34 p. Disponível em: https:// bdm.unb.br/bitstream/10483/23310/1/2018_ IsadoraDeMendoncaRibeiroPereira_tcc.pdf

22. Almeida RCAA, Castro JM, Oliveira TVC, et al. Cobertura vacinal ANTI-HPV e motivos de não vacinação. REAEnf. 2020;2:e2600. doi: https://doi. org/10.25248/reaenf.e2600.2020

23. Brito-Silva K, Bezerra AFB, Chaves LDP, et al. Integralidade no cuidado ao câncer do colo do útero: avaliação do acesso. Rev Saúde Pública. 2014 Apr;48(2):240-8. doi: https://doi.org/10.1590/S00348910.2014048004852

24. Ribeiro L, Bastos RR, Vieira MT, et al. Rastreamento oportunístico versus perdas de oportunidade: não realização do exame de Papanicolaou entre mulheres que frequentaram o pré-natal. Cad Saúde Pública. 2016;32(6):e00001415. doi: https://doi. org/10.1590/0102-311X00001415

25. Viana JN, Moysés RPC, Espir TT, et al. Determinantes sociais da saúde e prevenção secundária do câncer do colo do útero no Estado do Amazonas, Brasil. Medicina (Ribeirão Preto). 2019;52(2):110-2. doi: https://doi. org/10.11606/issn.2176-7262.v52i2p110-120

26. Ribeiro CM, Silva GA. Avaliação da produção de procedimentos da linha de cuidado do câncer do colo do útero no Sistema Único de Saúde do Brasil em 2015. Epidemiol Serv Saúde. 2018;27(1):e20172124. doi: https://doi.org/10.5123/s1679-49742018000100004

27. Ministério da Saúde. Manual técnico operacional SIA/ SUS: sistema de informaçôes ambulatoriais: orientações técnicas [Internet]. Brasília, DF: Ministério da Saúde; 2010 mar [acesso fev 5]. Disponível em: http:// w3.datasus.gov.br/sia/index.php?area $=0401$

28. Scatena JHG, Tanaka OY. Utilização do Sistema de Informaçóes Hospitalares (SIH-SUS) e do Sistema de Informaçóes Ambulatoriais (SIA-SUS) na análise da descentralização da saúde em Mato Grosso. Inf Epidemiol Sus. 2001 mar;10(1):19-30. doi: http://doi.org/10.5123/ S0104-16732001000100003 